\title{
EXPLORING MANAGEMENT OF ARRIVAL SPACING USING ROUTE EXTENSIONS WITH TERMINAL SPACING TOOLS
}

\author{
Bonny Parke, Nancy Bienert, Eric Chevalley, Faisal Omar, Nathan Buckley, Connie Brasil, Hyo-Sang \\ Yoo, Abhay Borade, Conrad Gabriel, San Jose State University Research Foundation/NASA Ames, \\ Moffett Field, $C A$
}

Paul Lee, Jeff Homola, Nancy Smith, NASA Ames Research Center, Moffett Field, CA

\begin{abstract}
Airports with shared runway operations between arrivals and departures can experience severe departure gridlock and delays during a heavy arrival push due to insufficient gaps in the arrival stream for aircraft to depart. The problem is accentuated in situations when a large gap in the arrival spacing has to be created at the last minute due to wake vortex separation requirements. At LaGuardia airport, wake vortex separation problems arise when a heavy jet, such as a B757, departing on Runway 31 needs additional spacing between arrivals on Runway 22 .
\end{abstract}

A standard solution for controllers in many airports in situations such as this is to extend the downwind leg of arrival aircraft to create extra space between the arrivals. The question addressed in this paper is how such route extensions would work with terminal scheduling operations, namely (1) the automated Terminal Sequencing and Spacing (TSS) tools and (2) a new scheduling tool which increases the availability of gaps for departure aircraft (Departure Sensitive Arrival Spacing or DSAS). In a simulated LaGuardia airport (LGA) Terminal Radar Approach Control (TRACON) airspace, two new RNAV arrival routes were created along with extensions to these routes. The arrival route from the south had a downwind leg extension near the airport in the final sector. The arrival route from the north had an extension in a feeder sector further from the airport. An exploratory one-hour run with the route extensions was compared to an hour run without the extensions. Topics included in the paper are 1) how the route extensions were developed, 2) a procedure outlining how the aircraft could be scheduled to the extensions and who would do it, and 3) the results of the exploratory run compared to the original run without the extensions. The results indicated that the extended downwind leg route helped to create a B757 departure gap in the middle of a packed arrival stream, resulting in a reduction of 11 minutes in average wait time for the $\mathrm{B} 757 \mathrm{~s}$, but at a cost of increased controller self-reported workload from low to moderate.

\section{Introduction}

In recent years, NASA has developed a new concept called Terminal Sequencing and Spacing (TSS) to extend the existing Time-Based Flow Management (TBFM) tools in order to manage the arrival schedule to the runway threshold [1]. One potential issue with TSS and other terminal scheduling concepts is that the tactical adjustment schedule can be difficult as the aircraft nears the runway, resulting in a flow that becomes increasingly brittle [2][3]. If the arrival flow is packed, as it frequently is approaching the runway due to the decreased speed of aircraft ahead (compression), and one aircraft is not where it should be, it becomes difficult to maintain the required spacing between the other aircraft.

In this situation, controllers commonly extend the downwind leg to control spacing close to the runway. Figure 1 shows the typical landing pattern and the downwind leg in relation to the other legs and the runway [4].

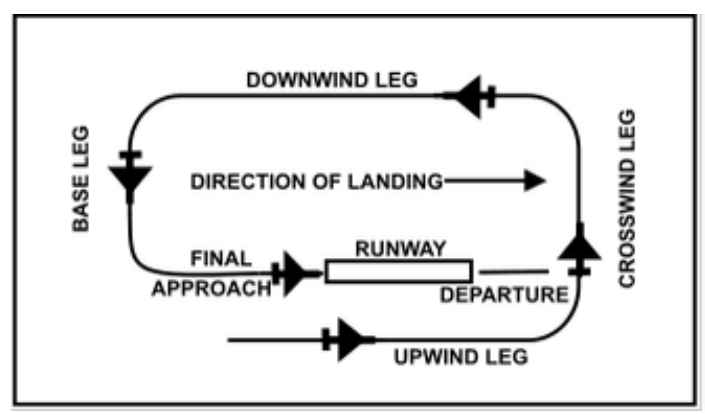

Figure 1. Typical Landing Pattern Showing Downwind Leg 
Routes further from the airport can also be extended to provide path options. Other researchers have tried to incorporate flexibility into terminal scheduling operations by adding extended or multiroute options to routes [5][6][7][8]. Varying degrees of automation have been involved. Recent research [9] has focused on creating a user interface which identifies the best turn point for the aircraft on the downwind leg, providing assistance to the controller on where the aircraft should begin its turn. This allows for a variety of downwind leg extension lengths and adaptations to specific spacing needs.

\section{Background}

A Human-in-the-Loop (HITL) simulation took place in August, 2014, which tested a new terminal operational concept called "Departure-Sensitive Arrival Spacing" (DSAS). DSAS allows for maximum departure throughput without adversely impacting the arrival traffic by adjusting the interarrival spacing slightly to create an optimal gap size for one or more departures [10]. This simulation demonstrated that the tools were helpful in creating the different sizes of gaps (Table 1) that were needed for LGA departures.

In the simulation, the controllers took two types of approaches to creating gaps for DSAS operations. The first type was a strategic gap creation in which the departure gaps were created in the arrival schedule prior to the TRACON entry, creating minimal workload for the TRACON controllers. A second type was a tactical gap creation for a particular departure aircraft type, such as a B757 departure during a 22|31 runway configuration (arriving on runway 22 and departing on runway 31 ). This required extra space between the arrivals for wake turbulence separation at the runway crossing and could be done only at the last minute when the B757 appeared in the departure queue.

In the high traffic run in this simulation with DSAS operations, the B757 departure was scheduled to depart in a natural gap too far upstream and had to wait for this gap to appear at the airport before departing. This resulted in being put on standby while 10 other departures left. This was deemed unacceptable by subject matter experts (SMEs) familiar with LaGuardia tower operations, even though it entailed a low controller workload to create the gap.

Table 1. Required Time for Departures

\begin{tabular}{|c|r|}
\hline $\begin{array}{c}\text { Type of } \\
\text { Departure }\end{array}$ & Seconds \\
\hline Single & 75 \\
\hline Double & 120 \\
\hline Triple & 170 \\
\hline B757 & 180 \\
\hline Quadruple & 220 \\
\hline
\end{tabular}

Although extending a downwind leg is a common practice for creating gaps, it was not part of the initial TSS and DSAS operations tested in the simulation, where controllers maintained the schedule mainly with speed adjustments along predefined RNAV routes. To enable controllers to create an extra-large arrival gap closer to the airport, exploratory runs tested route extensions designed to provide controllers with standardized path options, in addition to speed, to control the aircraft.

\section{Method}

\section{Simulated Airspace}

The simulated airspace consisted of a subset of the New York TRACON (N90) sectors that feed traffic into LGA Airport. Figure 2 shows the two feeder sectors (Empyr and Haarp) and one final sector (Final). These sectors have been modified from current sector configurations in order to accommodate optimized profile descent (OPD) routes that were newly designed for the simulation. 


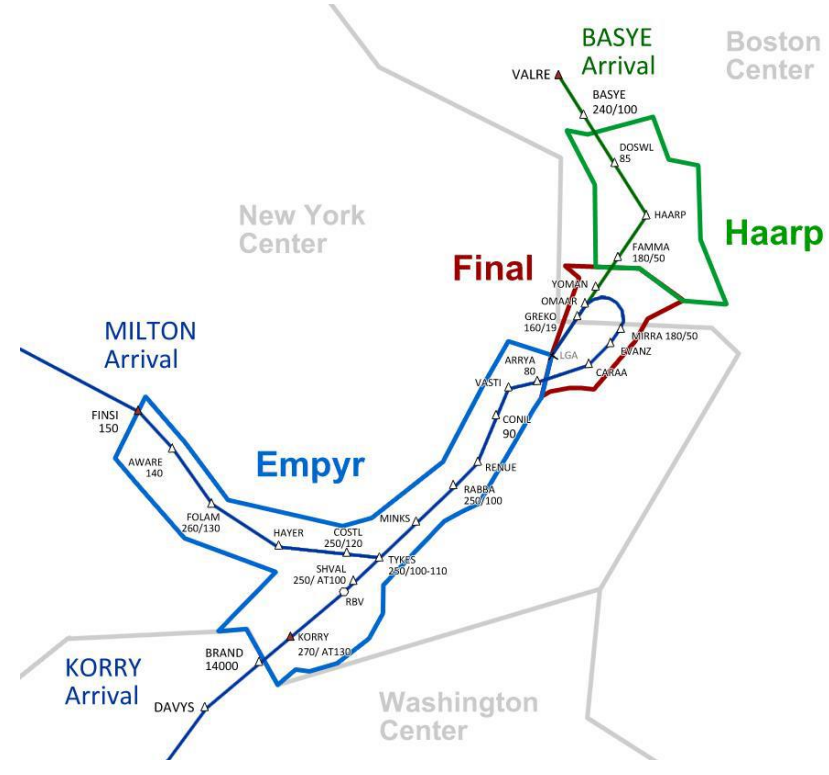

Figure 2. Simulated Airspace without Extensions

\section{Design of Route Extensions}

Figure 3 shows the two route extensions that were created (dashed lines). The southern downwind extension was designed to add 105 seconds at 180kts and was $5.25 \mathrm{~nm}$ longer than the original route. The increased time of 105 seconds was selected since a regular single departure slot was 75 seconds, as shown in Table 1. The difference between the single departure slot (75seconds) and a slot big enough for a B757 (180 seconds) is 105 seconds.

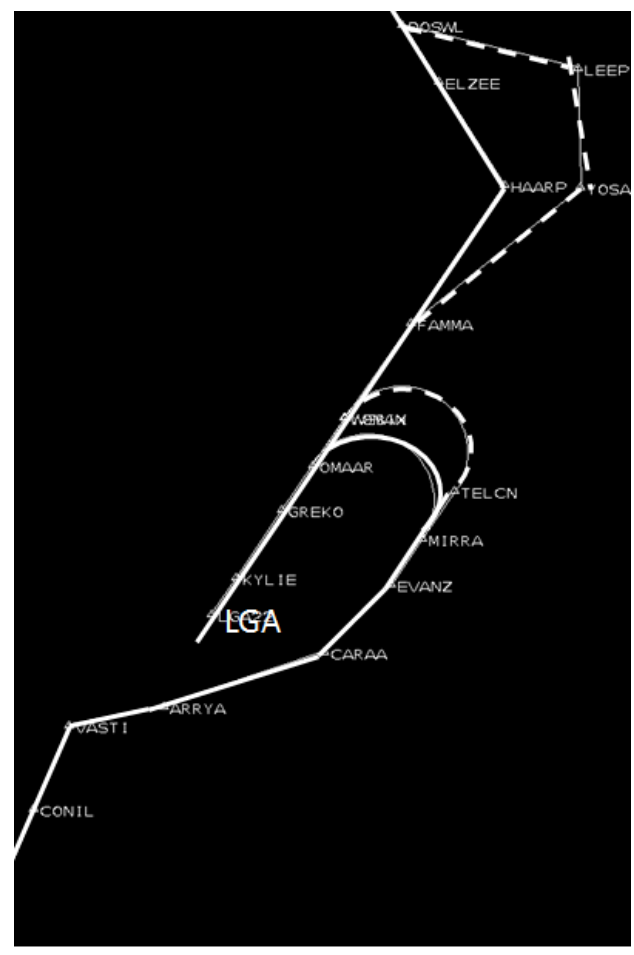

Figure 3. Two Route Extensions (Dashed)

The route extension coming from the north to LGA shown in Figure 3 was also designed to add 105 seconds. Its development was more complicated due to two reductions in speed over the two routes. The original route was designed to take 255 seconds from DOSWL to FAMMA. (The first $9.7 \mathrm{~nm}$ took 145 seconds at $240 \mathrm{kts}$ and the second $6.4 \mathrm{~nm}$ took 100 seconds at 210 knots). The extended route was designed to take 360 seconds from DOSWL to FAMMA. (The first $17.2 \mathrm{~nm}$ took 258 seconds at $240 \mathrm{kts}$ and the second $5.95 \mathrm{~nm}$ took 102 seconds at 210kts) As will be seen, the northern extension route followed the path of previous vectors the Haarp controller had made.

\section{Tools}

\section{Terminal Sequencing and Spacing Tools (TSS)}

Metering was based on the NASA Ames Traffic Management Adviser with Terminal Metering (TMA-TM) research [1][11]. The controller spacing tools (TSS) were based on the NASA Ames Controller Managed Spacing (CMS) research [12]. 
TSS was used to create and deliver an arrival schedule that would minimize the loss of departure slots due to inconsistent inter-arrival spacing. TSS enhances the existing meter fix scheduler to create a Terminal Metering schedule. Figure 4 shows an example of such a schedule for LGA in the 22|31 configuration. The right side of the graph shows the Scheduled Times of Arrival (STAs) to the Runway 22 threshold using wake vortex spacing under VFR conditions, which is greater than or equal to 75 seconds depending on the equipage types. The left side of the graph shows the Estimated Times of Arrival (ETAs). The numbers next to an aircraft's STA indicate the delay that the aircraft needs to absorb (in minutes) in order to conform to their STA. The wide spacing between "hj" (heavy jet) WJA1820 (third from bottom on right) and CHQ6962 (fourth from bottom on right) indicates a 180 second gap at the runway suitable for a heavy jet to depart. This is part of the new DSAS tool to be described later.

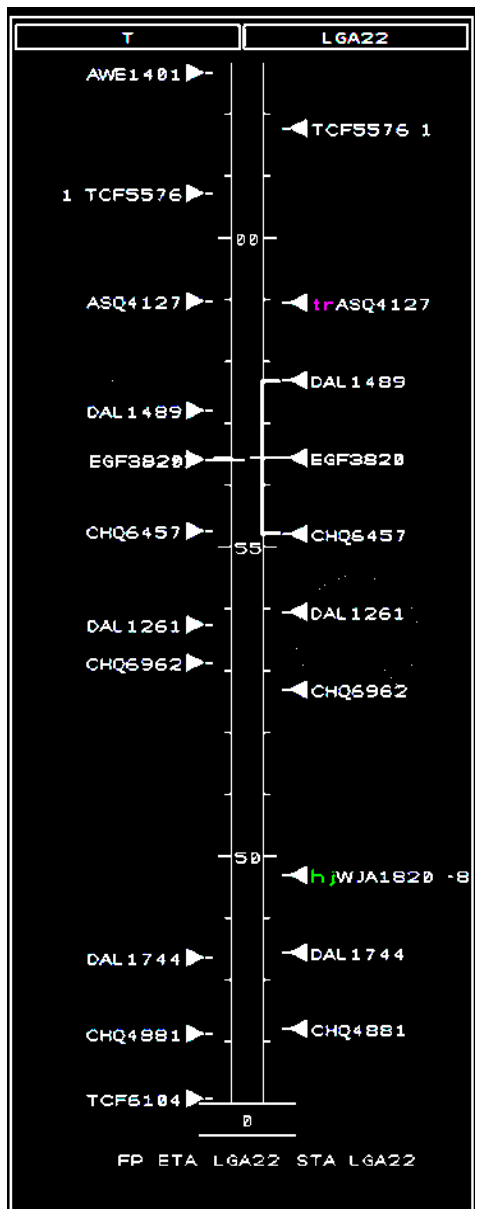

Figure 4. Example of TSS Metering Schedule for LGA

As shown in Figure 5, the TSS slot markers (yellow hollow circles) on the controller's scope indicate the ideal location for an aircraft to achieve conformance with its scheduled time of arrival or STA [13]. An indicated airspeed also appears next to the slot marker. In this figure, it can be seen that the Final controller also used bats (turquoise) to ensure that there would be sufficient separation from the aircraft ahead. The pointed end of the bat on the right in Figure 5 comes from CHQ6962 which was also depicted on the timeline in Figure 4 (fourth from the bottom on the right). The bat on this aircraft will help assure the right size of gap for the B757 which will depart between this aircraft and WJA1820, the aircraft ahead of it (also on the timeline in Figure 4). Figure 5 also shows that the aircraft is somewhat late and appears behind its slot marker. 


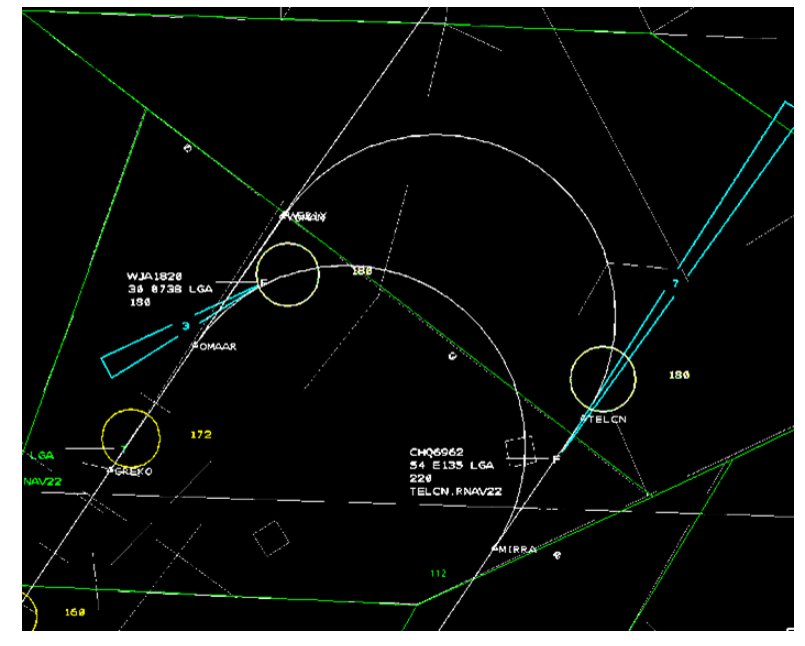

Figure 5. Example of TSS Slot Markers on Routes

\section{Departure-Sensitive Arrival Spacing Tool (DSAS)}

The DSAS concept assumed a new TMU Planner position (called "Planner" in the simulation), who could modify the schedule after the schedule was frozen and before the controllers started moving the aircraft to meet their STAs. We assumed that the aircraft were frozen at $150 \mathrm{~nm}$ from the airport and only the TRACON controllers were responsible for the STA conformance, giving the Planner about 20 minutes for adjusting the schedule to optimize the departures before the arrivals entered the TRACON.

The TSS was set to allow 75 seconds between arrivals - enough to allow one departure for each arrival. The Planner could adjust the schedule to allow more space between arrivals by using the DSAS spacing tool, shown in Figure 6. The top of Figure 6 shows DAL1046 and a bracket that indicated the minimum wake vortex spacing for that arrival. The green bars indicated the "slack capacity" that was in excess of the minimum inter-arrival spacing that was needed. The middle of Figure 6 shows the options presented to the Planner when the Planner right clicked on the call sign, (e.g., WV for normal wake vortex spacing, Double for spacing needed for two departures, Triple for three departures, etc.). When the Planner hovered over the item "Double" on the menu, the bracket for DAL1046 became larger to preview the spacing needed for two departures.
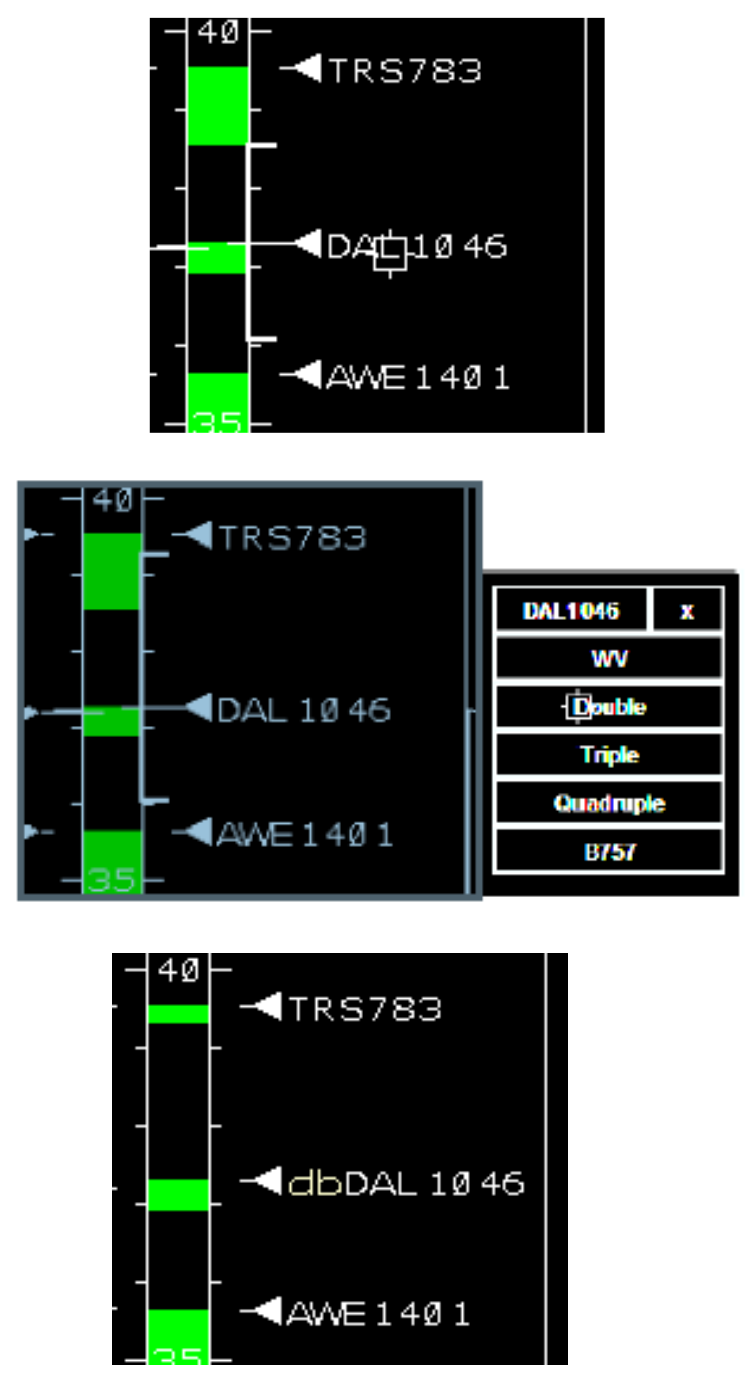

\section{Figure 6. Planner's DSAS tools [10]}

As shown on the bottom figure, when the Planner clicked on the "Double" spacing, the word "db" for double appeared next to the call sign DAL1046 to indicate that the larger spacing had been reserved for two departures and the green bar showed less slack capacity near the TRS78. The updated schedule and the "db" assignment were then propagated throughout the system to show the updated schedule to the impacted controllers. For the TRACON controllers, their STA conformance task remained the same, i.e., to try to put aircraft in their sector in the slot markers. 


\section{Route Extension Menu}

Figure 7 shows the Route Extension menu on the scope of the Empyr controller as he clicked the top TELCN route (red concentric circles). By clicking next on the datatag of the aircraft, he updated the ground system and assigned the aircraft to the extended route.

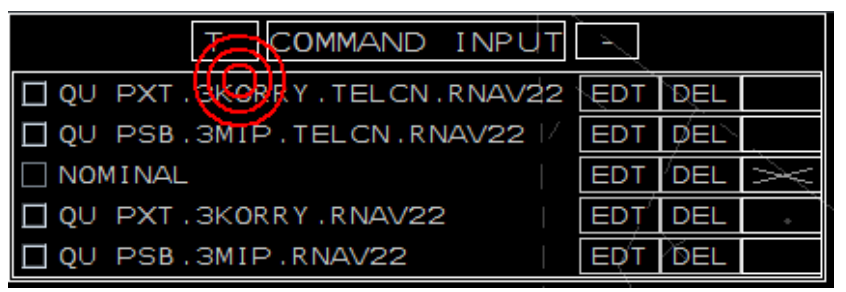

Figure 7. Route Extension Menu

\section{Experimental Design}

An exploratory run with the route extensions was compared to a run without the extensions. ${ }^{1}$ Both runs were in high traffic of around 40 aircraft per hour, which matches the nominal LGA Airport Arrival Rate (AAR) during peak traffic periods. Three highly experienced TRACON controllers handled the two feeder sectors and the final sector, with four other controllers in support positions (Center and Tower). Other experienced supervisory personnel in the simulation were a Planner (TMU confederate), an En Route Frontline Manager, and a TRACON Sequencer. The original run and the exploratory run had the same scenario with changed aircraft call signs. The exploratory run with the extended routes was preceded by three practice runs. There were no winds in the simulation. More detailed descriptions of the August, 2014, simulation can be found in [10].

\footnotetext{
${ }^{1}$ Although there were two exploratory runs to test the route extensions, the results of only one exploratory run are included in this paper since it was found that there was a software error in one run that affected almost all results. This error caused the aircraft flying the extended route to fly 56kts faster than those on the original route, when measured just before the extended route began. This increased speed reduced the time aircraft took to fly the extended route, and hence reduced the size of the gaps in the arrival stream.
}

\section{Apparatus}

Multi-Aircraft Control System (MACS) software was used to emulate Standard Terminal Automation Replacement System (STARS) displays shown on large-format monitors similar to those used in current air traffic control facilities. Keyboards similar to those used in the field further helped to replicate the look and feel of these facilities. MACS software provides a high fidelity environment in which to prototype scheduling tools, to simulate the air traffic, and to collect data [11]. In addition to STARS displays, MACS software was used to prototype Planner, Tower, and pseudo-pilot stations.

\section{Procedures}

\section{DSAS Procedure to Create Gaps}

The coordination developed for the DepartureSensitive Arrival Spacing tool (DSAS) in the 2014 simulation was followed [10]. When a B757 reached at least the seventh spot in the departure queue at the airport, the Cabin Coordinator in the tower called the TRACON Sequencer who coordinated with the two feeder controllers to determine the best location in the arrival stream to build a large enough gap. Once the location was agreed upon, the Sequencer called the Planner who then, with the aid of the DSAS tool, created the gap in the STAs on the TBFM timeline. This caused all of the aircraft STAs on the timeline that were in back of the desired gap to move backwards to incorporate the needed delay. This procedure was called "rippling the list." At the controllers' stations, all the slot markers, which were based on the STAs, also moved back behind the newly created gap. This change is depicted in Figure 8 and Figure 9. The controllers could then use speeds or vectors to position the aircraft in the new slot marker positions.

In the extended route condition, if there was too much delay, the controllers could use a new option, one which incorporated extension routes into the delay calculation.

\section{Procedure for Using Extended Routes to Absorb Excess Delays}

With this option, whenever the new slot marker position was too far behind the aircraft to use speed control to delay the aircraft, the extended route could be assigned to the aircraft, effectively moving the slot 
marker forward, closer to the aircraft. This involved the controller updating the system by using the Route Extension menu, shown in Figure 7. The TSS then incorporated the delay to be absorbed to later in the extended route. For the controller, all that remained was to clear the pilot to fly the extended route.

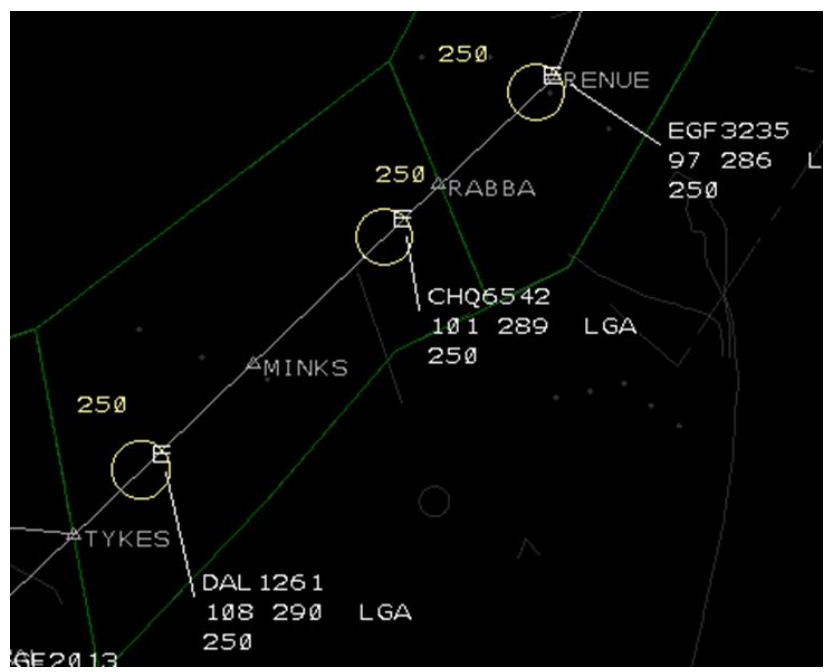

Figure 8. Aircraft in Slot Markers Before Ripple

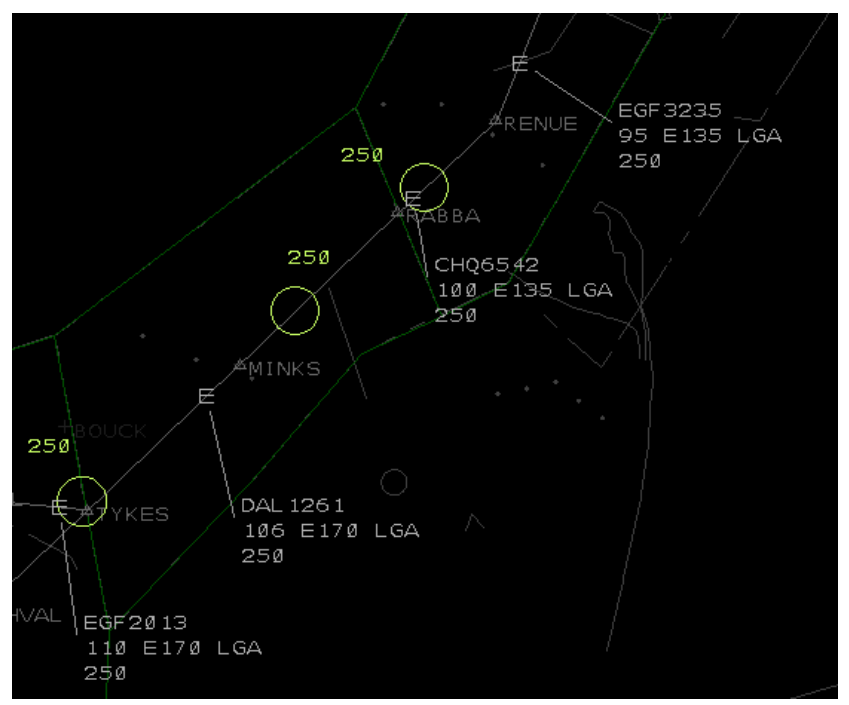

Figure 9. Aircraft Ahead of Slot Markers After Ripple

\section{Procedure to Return to Normal Operations}

Once the extended route option had been successfully used to create a gap allowing the B757 to depart, the controllers looked for an opportunity to put the subsequent arrivals back on the original, nominal route in order to shorten the flight distance and to enable the route extension to be used again, if needed. A gap is needed for this procedure as well, since without a large enough gap, the arrival aircraft being put on the nominal route will come too close to the aircraft ahead of it (still on the extended route where the extended route rejoins the nominal route). The procedure that the controllers developed involved looking ahead for a triplet of aircraft with a northern arrival in the middle which would then be vectored to make the necessary gap (105 seconds). The TRACON Sequencer looked on the timeline for such triplets and coordinated with the Empyr and Haarp controllers to execute the plan.

\section{Results}

\section{Experimental Results}

\section{Use of Extended Routes}

Table 2 shows that about $40 \%(17 / 43)$ of the eligible aircraft in the exploratory run were put on the extended routes. It should be noted, however, that when the northern feeder controller (Haarp) put an aircraft on the LEEPO extension, he usually vectored in addition (4/5 times).

Table 2. Number of Aircraft on Extended Routes

\begin{tabular}{|l|c|c|c|}
\hline $\begin{array}{c}\text { Sectors } \\
\text { with } \\
\text { Extended } \\
\text { Routes }\end{array}$ & $\begin{array}{c}\text { \# of Eligible } \\
\text { Aircraft on } \\
\text { Extended } \\
\text { Route }\end{array}$ & $\begin{array}{c}\text { \# of Eligible } \\
\text { Aircraft on } \\
\text { Nominal } \\
\text { Route }\end{array}$ & Total \\
\hline $\begin{array}{l}\text { Haarp } \\
\text { (LEEPO) }\end{array}$ & 5 & 6 & 11 \\
\hline $\begin{array}{l}\text { Final } \\
\text { (TELCN) }\end{array}$ & 12 & 20 & 32 \\
\hline Total & 17 & 26 & 43 \\
\hline
\end{tabular}




\section{Aircraft Tracks}

It appears that vectoring was not reduced for any of the three controllers, judging from the aircraft tracks in both conditions shown in Figure 10. The original route condition is on the left and the extended route condition is on the right. As can be seen, there is substantial vectoring in the Haarp northern feeder sector (green tracks) in both conditions, but especially in the extended route condition. The lack of vectoring in the original, nonextended route condition is most likely the result of placing the $\mathrm{B} 757 \mathrm{~s}$ on lengthy standbys to minimize their impact on traffic.

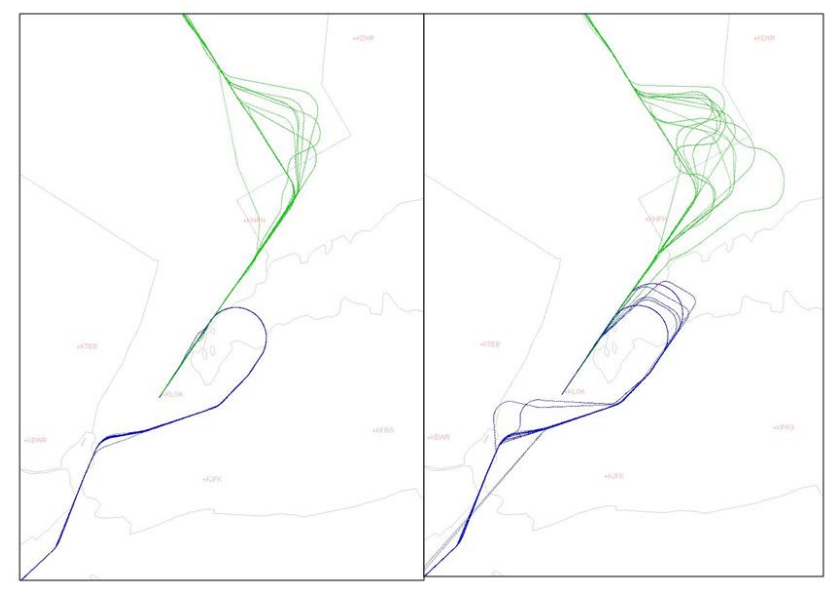

Figure 10. Original (L) \& Extended Route (R) Tracks

\section{Minutes in Queue for the B757s}

Table 3 indicates that the purpose of adding the route extensions was accomplished: the two B757s in each run departed earlier when there were extended route options for the controllers to assist in spacing arrivals nearer the airport. As can be seen in Table 3, the time the B757s were in the queue on the airport surface was reduced by an average of about 11 minutes with the extended route options.
Table 3. Minutes in Queue for B757s

\begin{tabular}{|l|r|r|}
\hline & Original & Extended \\
\hline B757 \#1 & 26.42 & 10.07 \\
\hline B757 \#2 & 18.22 & 12.20 \\
\hline Average & 22.32 & 11.14 \\
\hline
\end{tabular}

\section{Arrival and Departure Rates}

Table 4 indicates that the reduction in B757 queue time was accomplished without a major impact on the numbers of arrivals and departures in the onehour runs.

Table 4. Number of Arrivals and Departures

\begin{tabular}{|l|c|c|}
\hline Aircraft/hr. & Original & Extended \\
\hline Arrivals & 40 & 43 \\
\hline Departures & 47 & 46 \\
\hline Total & 87 & 89 \\
\hline
\end{tabular}

\section{Other Sizes of Gaps Created}

Route extensions should help with the creation of all types of gaps, not just the B757 gaps. The types of increased spacing created in the two runs are shown in Table 5. Although it is hard to compare the types, there were slightly more gaps created with the route extensions than without.

Table 5. Types of Increased Spacing

\begin{tabular}{|c|c|c|r|}
\hline & Original & Extended & Totals \\
\hline $\begin{array}{c}\text { Wake } \\
\text { Vortex } \\
\text { Spacing }\end{array}$ & 2 & 1 & 3 \\
\hline Doubles & 6 & 10 & 16 \\
\hline Triples & 4 & 5 & 9 \\
\hline Quadruples & 3 & 1 & 4 \\
\hline \# of 757s & 2 & 2 & 4 \\
\hline Totals & 17 & 19 & 36 \\
\hline
\end{tabular}




\section{Safety}

There was no loss of separation in either run.

\section{Workload: Number of Clearances}

It can be seen in Table 6 that the number of clearances in the extended route condition was markedly higher than in the original condition, without the extensions. This increase is greatest for Final, going from a total of 6 clearances to 76 . In the extended route condition, the controllers tried to put the B757s earlier, closer to the airport, which caused excess delays for 10 or so subsequent arrivals. This did not occur in the original condition and could be one reason for the larger number of clearances in that condition.

Table 6. Clearances Per Sector Per Run

\begin{tabular}{|l|r|r|r|}
\hline Sectors & Original & Extended & Totals \\
\hline Final & 6 & 76 & 82 \\
\hline Empyr & 74 & 144 & 218 \\
\hline Haarp & 63 & 91 & 154 \\
\hline Total & 143 & 311 & 454 \\
\hline
\end{tabular}

Table 7 shows that the biggest increase for Final were speed clearances (from 6 to 76, over a 10-fold increase). Also, in the original run, Final had no altitude, direct-to or heading clearances and had 11 , 10 and 5 respectively in the extended route condition. Empyr also had an increase in altitude clearances.

Table 7. Types of Clearances per Sector per Run

\begin{tabular}{|c|r|r|r|r|r|r|}
\hline Sector & \multicolumn{2}{|c|}{ Final } & \multicolumn{2}{c|}{ Empyr } & \multicolumn{2}{c|}{ Haarp } \\
\hline Condition & Orig. & Ext. & Orig. & Ext. & Orig. & Ext. \\
\hline Speed & 6 & 50 & 71 & 107 & 44 & 45 \\
\hline Heading & & 11 & & 6 & 18 & 29 \\
\hline Altitude & & 10 & & 10 & & \\
\hline Direct-to & & 5 & 3 & 3 & 1 & 11 \\
\hline $\begin{array}{c}\text { Route } \\
\text { Change }\end{array}$ & & & & & & \\
\hline Totals & 6 & 76 & 74 & 144 & 63 & 91 \\
\hline
\end{tabular}

To ascertain whether the increase in clearances could be due to other factors, we compared the average time the arriving aircraft were off-schedule as they entered the TRACON in the two conditions.
We found that the average off-schedule time was minus 30.1 seconds in the original route condition, and minus 74.5 seconds in the extended route condition, $t(81)=3.4, \quad p=.001)$. Hence at the TRACON boundary, the delay to absorb was significantly greater in the extended route condition than in the original condition. This is another reason for the larger number of clearances in the extended route condition.

\section{Participant Assessments}

The participants were asked to fill out an on-line survey after each run and also participated in debriefs.

Safety

Figure 11 indicates that safety was deemed acceptable in all three sectors by all three controllers-both in the original and extended runs.

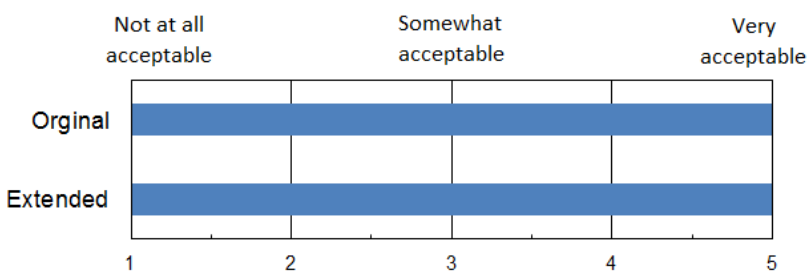

Figure 11. Ratings on Acceptability of Safety

\section{Workload and Acceptability of Workload}

As shown in Figure 12, the three controllers rated their mental activity during the busiest time in their sector as very low in the original run and more than moderate in the extended run (between 3 and 4 on a 5 point scale). The TRACON Sequencer also worked harder in the extended condition, with ratings of 2 and 5 in the two conditions. The Planner's ratings were 3 and 3 .

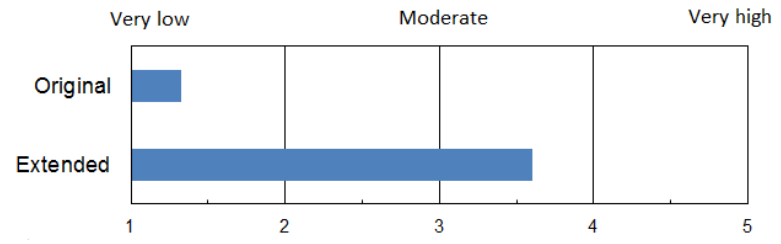

Figure 12. Ratings on Amount of Mental Activity 
As shown in Figure 13 below, the controllers' average ratings of workload was low in the original condition ( 2 on a 1 to 5 scale) and moderate (3) in the extended route condition. (Sequencer's ratings were 2 and 3, Planner's ratings were 3 and 3.)

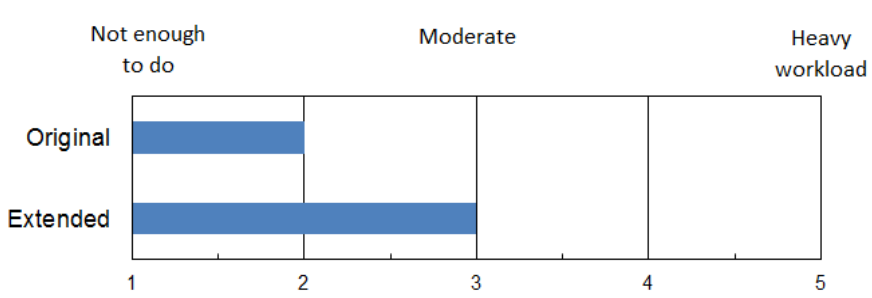

Figure 13. Ratings on Workload

The controllers rated the acceptability of workload as 5's in the original condition and an average of 4.7 in the extended condition (Empyr rated it as a 4.) The Sequencer's ratings were 5 and 4.

\section{Coordination}

The acceptability of coordination in both conditions was rated as 5 's by all three controllers. The Sequencer, however, rated it only as a 3, "Somewhat acceptable," stating, "Too much coordination with ripples and monitoring their speed adjustment." He recommended fewer ripples "when providing gaps where we have a double slot already." The Planner wrote that the coordination between the Planner and Sequencer "worked very well" in both conditions.

The controllers were asked after each run, "How many times there was a schedule change that affected you?" Figure 14 indicates that this schedule change (ripple) occurred more frequently in the extended route condition. In the extended route condition, the highest rating was 5-6 times for the Final controller, followed by Empyr 3-4 times, and then Haarp with 12 times.

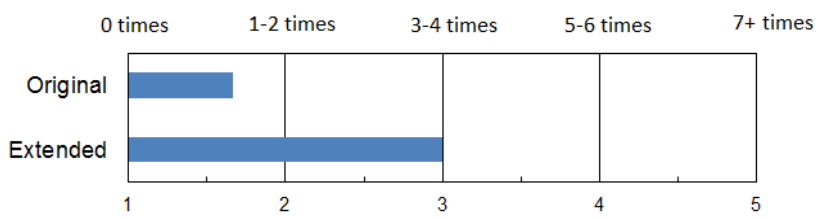

Figure 14. Reported Times Schedule Changed (Rippled)
The controllers were also asked "If there were any schedule changes that affected you, were you informed about them in advance?" All controllers indicated that they were "Always" informed about them in advance ( $5 \mathrm{~s}$ on a scale of 1 to 5$)$.

\section{Controller Assessment of the Tools and Procedures}

In general, the controllers were pleased with the extended routes and with their integration into the terminal and sequencing and spacing tools.

In the post-run survey they wrote the following comments.

Empyr: "The extended routes worked well because they eliminated my need to vector. Once I put them in, the delay was such that speeds were enough to fix the sequence."

Haarp: "The extended route worked very well when the aircraft was about 7-9 miles in front of the slot marker. If the aircraft is more or less than 7-9 from the slot markers, it's too much vectoring and very little airspace to hit the slot markers."

TRACON Sequencer: "The routes worked great and really assisted the Empyr controller in his sequences."

Planner: "It appeared much easier for the controllers to use the extended route. They seemed to get into it and out of it fairly well. Another good tool for the toolbox."

In the debrief, more aspects of the tools and the simulation were discussed. First, in the extended route run, the Planner said he "thought it would be a good exercise to give the TRACON a lot of big [delay] numbers, [to see] how they handled it with the extended routes. .. And to me, they were getting big numbers at the meter fixes and then by the time they were touching down, they were all pretty much spot on for what [Final] was delivering to the tower. I thought it went well."

Empyr responded, "I agree, the guys came in with larger than normal delays. . When I did use the long route tool, it made the job real easy. Basically it took a delay that I would have done some vectoring for and changed it to a delay where now speed is going to take care of it. . . . I can absorb definitely three minutes just with speed." 
Haarp stated that if the markers "are about 10 miles behind the airplane, the Leepo [extended route] works really well, other than that, it is better to just leave it on the normal route and vector it to get back in the slots." Haarp suggested varying the sizes of extended routes to match the needed delay.

A problem with the tools that emerged in the debrief was that they resulted in much speeding aircraft up and then slowing them down, which, in addition to increasing the controllers' workload, would adversely affect the pilots. A Subject Matter Expert (SME) from LGA noted that current day practices at LGA in such a situation would have involved holding at two fixes, probably a NY hold at Washington and a hold at Boston. And indeed, he thought that would have been preferable to what happened in the simulation where Empyr had the aircraft "all set up and it looked like it was going well, and then we rippled, and then even on Final [the slot markers] jumped back, and I think in reality, I might have spun the guy from Haarp, let him hold for maybe 10 minutes, and then let Empyr's traffic just come up. . . It just seemed that maybe delaying the one airplane would have resolved some of the speed control that Empyr needed at that point."

Empyr responded, "I guess the choice put [on] the pilots is that you either speed up and slow down, or you hold. They'd probably pick the former."

When asked if he would use the extended route option in the field given large delays on the arrivals coming in, Empyr responded, "I would use it. If I had that tool in the field, it would make it so I wouldn't have to vector and didn't have to do more work-absolutely, I would use it every time."

\section{Discussion}

Given that the Planner said he increased the delays on the aircraft in the exploratory run, and that the off-schedule data on aircraft entering the TRACON confirms this, it seems that the runs compared were not identical in an important respect. This certainly would account for part of the increase in clearances in the extended route condition, along with the increased clearances caused by inserting a large gap close to the runway. Nonetheless, it appeared that the extended routes and their integration with TSS and DSAS worked well in some situations. Empyr, the feeder controller that spaced planes for Final, found the tool very helpful and this was confirmed by the TRACON Sequencer. In areas further from the runway such as Haarp, where there is space to vector, it might be advantageous to have extended routes of various sizes to meet the various delay needs.

In general, putting aircraft on alternate routes instead of vectoring them has many advantages, as described by Bienert, et al. [5]:

1. the controller gives one clearance to the flight deck instead of two or three (to initiate, turn, and finish the vector),

2. the controller doesn't have to monitor the position of the aircraft as much as during vectoring,

3. where the aircraft is going is written in the aircraft datatag for all to see, reducing the need for coordination and communication between controllers,

4. radio communication is reduced, and

5. the pilot can keep the navigation system engaged.

A final advantage over vectoring is that alternate routes enable adjustments in TBFM schedules, as demonstrated in this paper. This is especially useful near the runway. Alternate routes could also help in mixed equipage operations by separating equipped and unequipped aircraft, such as aircraft that can and cannot make tight RNP turns. Integrating extended routes into TBFM procedures is therefore relevant for many NextGen initiatives.

\section{Summary and Conclusion}

An earlier simulation showed a long wait time on the airport movement area for a B757 to depart from LGA in a condition with TBFM tools (TSS) and DSAS (a new tool to space arrivals based on departure needs). To reduce this wait time, we developed a downwind leg extension near the runway and an extended route further from the airport. We also developed the procedures necessary to integrate the route extensions into TBFM tools (TSS) and in DSAS. We then ran a one-hour simulation to test the effectiveness of these routes, and compared this exploratory run with a run that had the same tools but no extensions. 
We found that in the exploratory run, the controllers used the two extended routes about $40 \%$ of the time. The extended routes were successful in enabling the creation of a B757 gap in the middle of a packed arrival stream, reducing the average wait time for the B757s by about 11 minutes. Arrival and departure rates between the two runs were similar, as were the total number of other types of gaps created. There was no loss of separation in the two runs, and the controllers judged both runs as acceptable in terms of safety. However, aircraft tracks and clearance information showed that the extended routes did not reduce vectoring-if anything vectoring increased. Indeed, the total number of clearances increased, especially for Final, although the workload was judged as moderate and as acceptable. Two reasons for this increase in vectoring and clearances in the exploratory run are likely: 1) larger delays needed to be absorbed by large gaps being inserted into the arrival stream close to the runway, and 2) larger delays needed to be absorbed when aircraft first entered the TRACON due to an over-active confederate. The latter finding was unexpected and suggests that the increase in clearances observed in this exploratory run would be lower in reality.

The extended downwind route close to the runway was the most successful, helping the feeder controller Empyr space the arrivals for Final. When the Empyr controller was asked if he would use this tool in the field, he responded, if "I wouldn't have to vector and didn't have to do more work-absolutely, I would use it every time." The Sequencer confirmed its usefulness. The Planner said that even though he had created bigger delays coming into the TRACON, the aircraft "were all pretty much spot on for what [Final] was delivering to the tower. I thought it went well." The feeder controller Haarp who used an extended route further from the runway, stated that the extension was useful but only for delays of about ten miles and suggested that there be more extended route options tailored to the amount of delay that needed to be absorbed.

A disadvantage of changing the scheduled time of arrival (rippling the list) was that it resulted in controllers having to speed aircraft up and then, possibly later, slow them down. The extended route was designed to alleviate this problem, but did not completely do so. Other options for creating space near the runway also have trade-offs in controller/pilot workload, efficiency, and fuel burn.

A more thorough investigation of extended routes and NextGen tools seems warranted since the extended routes provide a needed flexibility to TBFM near the runway, could facilitate handling different levels of aircraft equipage, and since the controllers found them useful. We hope that this exploratory run provides a starting point.

\section{References}

[1] Thipphavong, J., J. Jung, H. Swenson, L. Martin, M. Lin, J. Nguyen, 2013, Evaluation of the Terminal Sequencing and Spacing System for Performance-Based Navigation Arrivals, Proceedings of the 32nd Digital Avionics Systems Conference, Syracuse, NY.

[2] Diffenderfer, P.A., T. Zheng, P. Gaea, 2013, Automated Integration of Arrival/Departure Schedules, Proceedings of the Tenth USA/Europe Air Traffic Management Seminar, Chicago, Illinois.

[3] Jung, J., S. Verma, S. Zelinski, T. Kozon,, L. Sturre, 2015, Assessing Resilience of Scheduled Performance-Based Navigation Arrival Operations, Proceedings of the 10th USA/Europe Air Traffic Management Research and Development Seminar (ATM2015), Lisbon, Portugal.

[4] FAA, 2014, Aeronautical Information Manual, Figure 4-3-1, 4-3-14, p. 187.

[5] Bienert, N., E. Chevalley, J. Kraut, B. Parke, E. Palmer, 2015, Effect of Using Multiple Route Options and the Conformance Tools to Deliver Aircraft to a Departure Fix, Presentation at the $6^{\text {th }}$ International Conference on Applied Human Factors and Ergonomics.

[6] Rein-Weston, D., R.H. Jacoby, E. Chevalley, A. Globus, H. Yoo, B. Parke, P.U. Lee, F.G. Omar, J. M. Kraut, N. Bienert, A.R. Borade, C. V. Gabriel, K.M. Gonter, E.A. Palmer, 2014, Development of a Route Crossing Tool for Shared Airspace Environments, Proceedings of the 33rd Digital Avionics Systems Conference, Colorado Springs, CO. 
[7] Parke, B., E. Chevalley, N. Bienert, P.U. Lee, K.M. Gonter, F.G. Omar, J.M. Kraut, H. Yoo, A.R. Borade, C. Gabriel, D. Rein-Weston, E. Palmer, 2015, The Effectiveness of a Route Crossing Tool in a Simulated New York Airspace, Proceedings of the Eleventh USA/Europe Air Traffic Management Research and Development Seminar, Lisbon, Portugal

[8] Zelinsky, S., J. Jung, 2015, Arrival Scheduling with Shortcut Path Options and Mixed Aircraft Performance, Proceedings of the Eleventh USA/Europe Air Traffic Management Research and Development Seminar, Lisbon, Portugal.

[9] Ohneiser, O., M. Temme, J. Rataj, 2015, Trawl-Net Technology for Timely Precise Air Traffic Controller Turn-to-Base Commands. Proceedings of the Eleventh USA/Europe Air Traffic Management Research and Development Seminar, Lisbon, Portugal.

[10] Lee, P.U., N.M. Smith, J. Homola, C. Brasil, N. Buckley, C. Cabrall, E. Chevalley, B. Parke, H. Yoo, 2015, Reducing Departure Delays at LaGuardia Airport with Departure-Sensitive Arrival Spacing (DSAS) Operations, Proceedings of the Eleventh USA/Europe Air Traffic Management Research and Development Seminar, Lisbon, Portugal.

[11] Swenson, H., J. Thipphavong, A. Sadovsky, L. Chen, C. Sullivan, L. Martin, 2011, Design and Evaluation of the Terminal Area Precision Scheduling and Spacing System, Proceedings of the 9th USA/Europe Air Traffic Management Research and Development Seminar, Berlin, Germany.

[12] Kupfer, M., T. Callantine, L. Martin, J. Mercer, Controller Support Tools for Schedule-Based Terminal-Area Operations, 2011, Proceedings of the 9th USA/Europe Air Traffic Management R\&D Seminar, Berlin, Germany.

[13] Prevot, T., P.U. Lee, T. Callantine, J. Mercer, J. Homola, N.M. Smith, E. Palmer, 2010, Human-inthe-Loop Evaluation of NextGen Concepts in the Airspace Operations Laboratory, Proceedings of the AIAA Modeling and Simulation Technologies Conference, AIAA 2010-7609, Toronto, Canada.

\section{Acknowledgements}

The authors acknowledge the skilled subject matter experts and controllers associated with this project.

\section{Email Addresses}

bonny.parke@nasa.gov

34th Digital Avionics Systems Conference

September 13-17, 2015 